Mots. Les langages du politique

\title{
La performance communicationnelle en contexte médiatique. L'exemple du débat politique télévisé
}

Guylaine Martel

\section{OpenEdition}

\section{Journals}

Édition électronique

URL : https://journals.openedition.org/mots/19532

DOI : $10.4000 /$ mots. 19532

ISSN : 1960-6001

Éditeur

ENS Éditions

\section{Édition imprimée}

Date de publication : 1 mai 2010

Pagination : 83-102

ISBN : 978-2-84788-209-4

ISSN : 0243-6450

Référence électronique

Guylaine Martel, « La performance communicationnelle en contexte médiatique. L'exemple du débat politique télévisé », Mots. Les langages du politique [En ligne], 92 | 2010, mis en ligne le 04 mai 2012, consulté le 23 avril 2022. URL : http://journals.openedition.org/mots/19532 ; DOI : https://doi.org/ $10.4000 /$ mots. 19532

\section{(C) ENS Éditions}




\section{La performance communicationnelle en contexte médiatique. L'exemple du débat politique télévisé}

Dans une perspective de recherche qui vise à reconnaitre et à développer des stratégies de communication efficaces pour la diffusion médiatique de l'information, le présent article aborde la question de la performance communicationnelle et, plus concrètement, la manière dont celle-ci s'accomplit dans le débat politique télévisé. La démarche méthodologique présentée dans la démonstration qui suit tend à expliquer pourquoi certaines organisations discursives seraient plus adéquates que d'autres pour transmettre ce type d'information en contexte d'interaction médiatisée. Si l'exemplification porte ici de façon plus restreinte sur le Débat des chefs canadien de 2004, la réflexion dans laquelle s'inscrit notre propos résulte d'une étude plus vaste portant sur 130 journaux télévisés québécois et 6 débats politiques diffusés au cours de récentes campagnes électorales canadiennes et québécoises ${ }^{1}$.

\section{Une approche interactionniste de la performance communicationnelle en contexte médiatique}

Alors que les modèles transmissionistes de la communication publique conçoivent la performance communicationnelle comme une représentation devant le public, l'approche interactionniste que nous proposons pour l'étude du débat politique télévisé s'inspire des modèles de construction du sens et suggère de définir la notion de performance en termes d'interaction avec le public. Dans cette perspective, la performance n'est pas considérée comme la construction exclusive de l'instance émettrice, mais comme le résultat de l'interaction entre les actants médiatiques et le public, une coconstruction empruntant à la fois aux espaces de production et aux espaces de réception de la communication.

Département d’information et de communication, Université Laval (Québec) Guylaine.Martel@com.ulaval.ca

1. Ce corpus représente près de 150 heures d'enregistrement. 
The lecturer and the audience join in affirming a single proposition. They join in affirming that organized talking can reflect, express, delineate, portray - if not come to grips with - the real world, and that, finally, there is a real, structured, somewhat unitary world out there to comprehend. (Goffman, 1981, p.194)

La démarche d'analyse que nous avons développée pour saisir les manifestations de la performance communicationnelle en contexte médiatique s’inspire de cette conception interactionniste, un positionnement qui s'avère profitable à plusieurs égards. D’abord, il réconcilie deux points de vue généralement présentés comme étant opposés et conduisant à des résultats nécessairement incomplets, soit l'étude de la performance abordée, ou bien sous l'angle de la production, ou bien sous l'angle de la réception. Selon la perspective interactionniste, tout locuteur acquiert au cours de ses diverses expériences interpersonnelles une compétence sociale à communiquer qui lui permet d'exploiter adéquatement l'ensemble des ressources du système langagier dans une situation donnée (Martel, 1998). Dans les échanges les plus quotidiens, la réussite communicationnelle d'un individu s'évalue en fonction de cette compétence à se comporter discursivement selon les standards établis et reconnus par sa communauté. Le même raisonnement s'applique à la communication médiatique. Les stratégies élaborées pour la télédiffusion du débat politique s'appuient sur les expériences médiatiques antérieures partagées par les médias et le public. Elles prennent en compte les critères de performance, bonne et mauvaise, admis par le public et les intègrent de manière à satisfaire ses attentes. Ainsi, que l'on se place du point de vue de la réception ou du point de vue de la production, la performance se construit et s’évalue en fonction de critères communs, socialement partagés.

Cette orientation du mot en fonction de l'interlocuteur a une grande importance. En fait, tout mot comporte deux faces. Il est déterminé tout autant par le fait qu'il procède de quelqu'un que par le fait qu'il est dirigé vers quelqu'un. Il constitue justement le produit de l'interaction du locuteur et de l'auditeur. Tout mot sert d'expression à l'un par rapport à l'autre. À travers le mot, je me définis par rapport à l'autre, c'est-à-dire, en dernière analyse, vis-à-vis de la collectivité. Le mot est une sorte de pont jeté entre moi et les autres. S'il prend appui sur moi à une extrémité, à l'autre extrémité il prend appui sur mon interlocuteur. Le mot est le territoire commun du locuteur et de l'interlocuteur. (Bakhtine, 1977, p.123-124)

L'approche interactionniste rappelle avec éloquence la participation active du public dans la coconstruction de la performance. Dans les termes de Goffman, le public est un «participant ratifié » (ratified participant)² de l'échange média-

2. Dans la théorie de Goffman, la notion de ratification renvoie aux divers rôles participatifs des interlocuteurs pendant l'interaction. Un interlocuteur ratifié correspond à un participant actif de l'interaction, en ce sens qu'il est manifeste que le locuteur s'adresse à lui et qu'il peut espérer de sa part une réaction. Selon Goffman (1981, p. 9-10) : «those ratified participants who are addressed, that is, oriented to by the speaker in a manner to suggest that his words are particularly for them, and that some answer is therefore anticipated from them. " 
tique. Bien sûr, l’interaction ne se réalise pas de la même manière en contexte médiatique qu'en contexte interpersonnel, mais le type de relation sociale qui s'établit entre le média et le public fait dire à Thompson que l'interaction médiatique, la quasi interaction selon son expression, n'est pas moins une forme d'interaction3.

It [mediated interaction] does not have the degree of reciprocity and interpersonal specificity of other forms of interaction, whether mediated or face-to-face. But mediated quasi-interaction is, none the less, a form of interaction. It creates a certain kind of social situation in which individuals are linked together in a process of communication and symbolic exchange. It is a structured situation in which some individuals are engaged primarily in producing symbolic forms for others who are not physically present, while others are involved primarily in receiving symbolic forms produced by others to whom they cannot respond, but with whom they can form bonds of friendship, affection or loyalty. (Thompson, 1995, p. 84-85)

Comme nos travaux précédents sur les journaux télévisés québécois ont permis de le montrer (Martel, 2007, 2008), la nouvelle télévisée s’inscrit le plus souvent dans une mise en scène interactionnelle qui vise précisément à simuler la présence du public dans l'espace médiatique 4 . Reproduisant les rituels de la conversation interpersonnelle et les caractéristiques du discours oral spontané, Hutchby constate que ces mises en scène rappellent la manière habituelle de communiquer des téléspectateurs dans leur propre espace communicationnel: "Broadcast talk is a form oftalk in public that, nevertheless, is oriented toward an approximation of the conditions of interpersonal communication in everyday face-to-face situations. » (2005, p. 411) 5

À l'instar de Tolson, pour qui les concepts de « interactivity, performativity, liveliness provide a fruitful starting point for the analysis of media talk» (2006, p.13), ily a tout lieu de croire que l'authenticité et le dynamisme des interactions simulées en contexte médiatique contribuent à la performance, dans la mesure où il s'agit de moyens qui encouragent la participation active du public. Poursuivant le raisonnement amorcé au début de cet article, on pourrait conclure de ce qui précède que la performance se définit en termes d'interaction et qu'elle s'accomplit dans les diverses stratégies de ratification du public.

[T]hough it is institutional, because of the way it is obliged to address its audience, media talk is also highly "conversational". It routinely makes use of the speech genres of ordinary conversation, including those mentioned by Bakhtine, but these are institutionally transformed and strategically manipulated by participants. Some of these genres include : stories, jokes, gossip, chat, witty repartee, banter, teasing

3. Voir la notion d'interaction parasociale dans Horton et Wohl, 1986, et l'ouvrage général de Fitch et Sanders, 2005.

4. Voir la double articulation du modèle de communication médiatique dans Isotalus, 1998. Une version adaptée de ce modèle pour les journaux télévisés se trouve dans Martel et Turbide, 2006.

5. Du côté francophone, voir les travaux de Relieu et Brock, 1995 et de Coulomb-Gully, 2001. 
and flirting, and of course selling and promoting all kinds of "products" (including oneself, as a "personality"). (Tolson, 2006, p.52)

Prenant en compte la nature et le contenu du message à communiquer, les canaux de diffusion qui sont sollicités et les caractéristiques des participants à l'interaction médiatique, on peut sans peine avancer que certaines stratégies de communication seront effectivement plus appropriées que d'autres pour ratifier le public, qu'une stratégie considérée comme étant efficace sous certaines conditions s'avérera totalement inopérante sous certaines autres. Par définition, un public non ratifié ne remplit pas son rôle de participant actif; il ne contribue pas de façon optimale à la coconstruction du message. Un cas extrême serait celui du téléspectateur qui ferme son poste de télévision ou change de chaine. Au contraire, une stratégie de ratification réussie prédispose le public à participer activement à la coconstruction du message. Faisant écho à la citation de Goffman au début de ce texte, la perspective de Bakhtine tend à valider l'importance de la ratification du public dans le processus de construction du sens.

La signification n'est pas dans le mot ni dans l'âme du locuteur, non plus que dans l'âme de l'interlocuteur. La signification est l'effet de l'interaction du locuteur et du récepteur, s'exerçant sur le matériau d'un complexe sonore donné. C'est l'étincelle électrique qui ne jaillit que lors du contact de deux pôles opposés. (Bakhtine, 1977, p. 146-147)

La piste de réflexion que nous avons suivie jusqu'ici nous amène à considérer la performance communicationnelle en contexte médiatique en fonction de sa capacité à ratifier le public dans une interaction qui favorise la construction du sens de l'information à transmettre. Une performance réussie serait celle qui prédispose le public à participer à la construction du sens du message. L'appréciation souvent assez intuitive que nous avons de la performance communicationnelle n'est pas sans lien avec l'impression de satisfaction qui nait d'une interaction où tous les participants ont le sentiment, sinon de s'être compris, du moins d'avoir conjointement produit du sens.

\section{L’analyse du discours et l'analyse de la conversation}

Telle qu'elle vient d'être présentée, l'approche interactionniste de la performance communicationnelle en contexte médiatique trouve un terrain de développement et d'exploitation privilégié dans l'analyse du discours et l'analyse de la conversation ${ }^{6}$. Pour ces deux courants linguistiques, les questions

6. Pour l'analyse du discours, voir Maingueneau, 1998; pour l'analyse conversationnelle, voir Drew et Heritage, 2006 et Vincent, 2001. 
concernant la nature et l'expression de la performance sont indissociables, cette dernière n'étant observable qu'à partir de ses manifestations en discours. De cette position, il faut comprendre que l'analyse fine des stratégies de ratification du public élaborées par les actants médiatiques en vue d'atteindre certains objectifs d'efficacité ne se limite pas à l'identification et à la description des procédés qui participent à la performance; elle révèle également les règles et les mécanismes par lesquels cette performance s'accomplit. Autrement dit, les stratégies qui ressortissent à l'analyse du discours et à l'analyse de la conversation du débat politique télévisé devraient permettre de remonter aux critères de performance en vue desquels elles ont été élaborées.

There is, however, a more fundamental methodological point on which CA [conversation analysis], in particular, insists. It is not just that there is a preference for "naturally occuring" and accurately transcribed data; it is also that this data, in principle, provides its own epistemological guarantees. (Tolson, 2006, p. 21)

Des analyses antérieures réalisées sur les journaux télévisés et les débats politiques ont permis de circonscrire trois composantes discursives en fonction desquelles seraient négociés les critères de performance en cours d'interaction : le genre télévisuel, l'identité professionnelle et l'identité personnelle des actants médiatiques. Chacune de ces composantes a déjà fait l'objet de descriptions détaillées auxquelles il convient de faire référence. Dans les limites de la présente étude, toutefois, nous insisterons plutôt sur le rôle de ces composantes à l'égard de la performance.

\section{Le genre télévisuel}

Le genre télévisuel7 renvoie au type de production médiatique : journal télévisé, débat, talk show ${ }^{8}$, etc. Malgré - ou peut-être en raison de - leur popularité, la description scientifique des genres télévisuels est assez récente9. D’une grande hétérogénéité discursive, la plupart d'entre eux résistent à une description stable. C'est précisément sur cette instabilité que repose, en partie du moins, la négociation des critères de performance au cours de l’interaction médiatique. En effet, si la négociation entre la production proposée

7. Voir Charaudeau $(1991,1997,2005)$ et Jost $(1998,2007)$.

8. Le talk show, et tout particulièrement les émissions de discussion mettant en scène des participants ordinaires venus témoigner de leurs expériences personnelles - Claire Lamarche (Québec), Oprah (États-Unis), C'est mon choix (France), etc. -, est certainement le genre télévisuel qui a reçu le plus d'attention de la part des analystes du discours et de la conversation au cours des dernières années.

9. Pour l'information télévisée, voir Charaudeau, 1997 et Martel, 2004. Pour le talk show, voir Charaudeau, 1991 et Tolson, 2001. Pour le discours politique télévisé, voir Charaudeau, 2005, Simons et Aghazarian, 1986 et Trognon et Larrue, 1994. Pour des types variés de discours médiatiques, voir Fairclaugh, 1995; Hutchby, 2005, 2006 ; Livingstone et Lunt, 1994 ; Scannell, 1991 ; Scollon, 1998; Thompson, 1995 ; Tolson, 2006 ; enfin, le numéro 37 de la revue Politix. 
par le média et le modèle que se représente le téléspectateur à partir de ses expériences télévisuelles antérieures ne débouche pas sur une sorte de compromis, la performance s'en trouve affectée. Si la production ne satisfait pas les contraintes du genre dont elle se réclame et qu'elle ne rejoint pas les attentes des téléspectateurs, le risque est grand pour qu'il y ait rupture du contrat de communication (Charaudeau, 1991). Même dans le cas où l'interaction est maintenue, la construction du sens du message souffre de ce que le public reconnait mal le cadre d'interprétation dans lequel on lui demande d'inscrire sa participation. Il ne comprend pas bien ce qu'on attend de lui. Cette ambigüité, que le téléspectateur ressent comme une sorte d'insatisfaction, joue très certainement sur sa perception de la performance ${ }^{10}$.

\section{L'identité professionnelle}

L'identité professionnelle ${ }^{11}$ renvoie au rôle assumé par les actants pendant la communication médiatique. Comme le genre télévisuel, l’identité professionnelle impose un certain nombre de contraintes quant à la manière de se comporter des actants médiatiques, contraintes qui font elles aussi l'objet d'une négociation au cours de l'interaction. Si l'actant ne se comporte pas conformément au rôle professionnel auquel il prétend - celui de chef du gouvernement, par exemple -, si sa manière de se présenter ne correspond pas aux attentes du public, à l'image que ce dernier se fait de la fonction sociale, l'interaction médiatique connaitra des ratés et la performance ne sera pas optimale. C'est d'autant plus vrai en ce qui concerne l'information politique, celle-ci étant intimement liée à la crédibilité des individus qui l'incarnent. Si le téléspectateur ne reconnait pas le cadre d'interprétation que représente la personnalité publique, s'il a des raisons de douter du sérieux, de la compétence ou de l'honnêteté du politicien, la construction du sens du message en sera affectée.

\section{L’identité personnelle}

Dans le cadre de la communication médiatique, l'identité personnelle ${ }^{12}$ correspond à ce qu'on pourrait appeler la valeur humaine ajoutée. Elle se dis-

10. Je ne réfère pas ici aux émissions de genre hybride comme Infoman ou Le fric show (Québec), Rick Mercer ou This hour has twenty-two minutes (Canada), lesquelles récupèrent des évènements de l'information sérieuse dans le cadre de productions humoristiques. L'expérience télévisuelle du public rend ce dernier compétent à coconstruire le sens de ces messages informatifs en fonction du contexte humoristique. On doit même considérer que la bonne performance communicationnelle de ces émissions tient précisément à la capacité du public de reconstruire le message à partir d'un cadre différent - ironique, très souvent - de celui dans lequel il a été présenté originalement.

11. Voir Burger, à paraitre, et Charaudeau et Maingueneau, 2002, pour la notion d'ethos préalable.

12. Voir Amossy, 1999, 2000 ; Kerbrat-Orecchioni et de Chanay, 2005. 
tingue de l'identité professionnelle en ce qu'elle renvoie à l'individu privé plutôt qu'à la fonction sociale qui motive généralement la présence de l'actant sur la scène médiatique. On croirait à tort, toutefois, que l'identité professionnelle justifie à elle seule la présence de l'actant dans l'espace médiatique et que l'identité personnelle est réservée à l'espace privé; en fait, l'image publique "combine the authenticity of the self with a professional, public role» (Tolson, 2006, p.162). Au rôle professionnel, l'identité personnelle ajoute à la négociation des éléments dont il résulte des personnalités perçues comme étant plus ou moins sympathiques, arrogantes, sages, agressives. En communication politique particulièrement, les efforts sont grands pour dissimuler l'identité professionnelle négative des politiciens ${ }^{13}$ derrière une identité personnelle plus favorable ${ }^{14}$. L'identité personnelle étant le lieu privilégié de l'expression du dynamisme et de l'authenticité, il n'est pas étonnant qu'on en retrouve des traces de plus en plus fréquentes dans les productions médiatiques qui cherchent à ratifier le public : "Elsewhere on TV and radio people perform not as actors, but as "themselves". Their performances then engage our attention as if they were conversations with which we could potentially interact. » (Tolson, 2006, p.13) En cohérence avec le genre télévisuel - le débat politique est moins tolérant à l'égard de l'expression de l'identité personnelle que le talk show -, le recours à l'identité personnelle est certainement une stratégie de ratification efficace, favorisant la performance communicationnelle.

Les contraintes et les attentes du genre télévisuel et des identités professionnelle et personnelle font l'objet d'une négociation constante entre la production médiatique et le public. Les chaines de télévision proposent des stratégies de ratification variées pour susciter la participation du public à ces interactions : "TV performers construct identities to engage with the audience. » (Tolson, 2006, p. 46) Pour ce faire, elles tendent à mettre en scène l'information dans un cadre de référence partagé par le public et selon un mode d'interaction qui se rapproche de la manière interpersonnelle de communiquer.

13. Selon les résultats d'un sondage Léger Marketing publié le 19 mars 2006 , les politiciens occupent le dernier rang des professions quant à la confiance accordée par le public.

14. L’identité personnelle des chefs d'antenne québécois est déterminante quant à la fidélisation des auditoires à un journal télévisé, et il n'est pas étonnant de voir que ces productions laissent de plus en plus de place à son expression à l'intérieur comme à l'extérieur du bulletin de nouvelles. Ainsi, le 22 Heures de TVA a doublé son temps d'antenne pour donner plus de place aux interactions entre les divers actants médiatiques et la présentatrice vedette, Sophie Thibault, récipiendaire de prestigieux prix du public depuis plusieurs années. Parallèlement, les recherches de Marie-Anne Laramée, 2003, révèlent qu'une partie de l'identité personnelle des journalistes de télévision se construit à l'extérieur des bulletins d'information, par l'intermédiaire des magazines populaires notamment. 


\section{L'analyse de la performance communicationnelle dans le débat politique télévisé}

Au cours de la campagne électorale fédérale de 2004, deux équipes de recherche, l'une spécialisée en analyse du discours médiatique (Lab-0)15 et l'autre en étude de la réception (GSR) ${ }^{16}$, ont mis leur expertise en commun pour réaliser une expérience visant à saisir sur le vif la perception d'électeurs québécois quant à la performance des politiciens pendant un débat télévisé. Elles ont réuni une trentaine de personnes, d'âge, de sexe et d'allégeance politique différents, et leur ont demandé d'évaluer, en temps réel et en continu17, la performance communicationnelle des chefs des quatre principaux partis politiques fédéraux pendant la diffusion en direct du plus important évènement médiatique de la campagne électorale : le Débat des chefs. À partir d'un appareil de contrôle individuel, chaque électeur avait pour consigne d'évaluer, sur une échelle de 1 à $100^{18}$, la performance des politiciens tout au long du débat. Chacun des appareils de contrôle était relié à un ordinateur permettant de présenter les résultats de l'évaluation de manière globale, individuelle, ou en fonction de l'un ou l'autre des paramètres retenus pour l'expérience, soit l'âge, le sexe et l'allégeance politique. Ainsi, la courbe blanche qui apparait sur chacune des photos qui accompagnent les extraits 1 à 5 représente l'évaluation moyenne produite par l'ensemble des 30 électeurs.

Mentionnons d'entrée de jeu que le résultat très faible obtenu au cours de cette évaluation est somme toute assez cohérent avec le genre télévisuel correspondant au débat - une émission d'information d'une durée de deux heures quinze, un contexte formel, statique et répétitif - et aux identités professionnelle et personnelle des débattants - des politiciens sérieux à la personnalité très sobre et au discours assez monotone. À partir d'un calcul, nécessairement approximatifi9, du nombre de mots, le tableau 1 indique que

15. Le Laboratoire de recherche sur les stratégies de communication à l'oral s'intéresse aux mises en scène élaborées par les médias audiovisuels pour la diffusion de l'information, principalement les journaux et débats politiques télévisés. Voir le site : www.com.ulaval.ca/lab-o (consulté le 26 novembre 2009).

16. Le Groupe de recherche en sécurité routière se spécialise dans l'étude des interventions visant la modification des comportements des usagers de la route. Ses travaux portent sur les méthodes d'évaluation de la réception en communication publique, plus particulièrement sur l'efficacité des campagnes de sécurité routière.

17. Cette méthode réfère à ce que Biocca, David et West, 1994, ont développé sous le nom de technique d'évaluation continue.

18. Une expérience similaire a été réalisée sur le Débat des chefs fédéral de 2008 à partir du même appareil, mais sur une échelle de 1 à 10 . Les résultats ne permettent pas de dire laquelle des deux mesures est la plus fiable.

19. En raison d'un décalage estimé à deux secondes entre la production discursive des chefs, le temps de réaction nécessaire aux électeurs pour l'évaluer et le temps d'enregistrement de l'appareil, il est impossible de délimiter au mot près la portion de discours visée par l'évaluation. 
plus de $91 \%$ de la production discursive des politiciens s'inscrit dans une zone d'évaluation ne dépassant pas $10 \%$. En fait, pour plus des deux tiers $(67,4 \%)$ de la production discursive du débat, l'évaluation moyenne de la performance communicationnelle des chefs n'a pas dépassé $5 \%$; à 26 occasions, pour $4,7 \%$ de la production discursive, l'évaluation s'est située entre $10 \%$ et $15 \%$; à 15 occasions, pour 2,9\% de la production discursive, elle s'est située entre $15 \%$ et $20 \%$; à 5 occasions, pour $1,3 \%$ de la production discursive, elle s'est située entre $20 \%$ et $25 \%$.

\begin{tabular}{rcc}
\hline $\begin{array}{r}\text { Évaluation moyenne } \\
\text { des électeurs } \\
\text { (à partir d'une échelle } \\
\text { graduée } \\
\text { de 0\% à 100\%) }\end{array}$ & $\begin{array}{c}\text { Fréquence des passages } \\
\text { discursifs considérés comme } \\
\text { étant les plus performants } \\
\text { (en nombre d'occurrences) }\end{array}$ & $\begin{array}{c}\text { Production discursive concernée } \\
\text { (calcul aproximatif réalisé } \\
\text { à partir du nombre de mots } \\
\text { correspondant à l'échelle } \\
\text { évaluative) }\end{array}$ \\
\hline $\begin{array}{r}\text { entre 0\% et 5\% } \\
\text { (évaluation la plus } \\
\text { faible) }\end{array}$ & - & $67,4 \%(13375 / 19857$ mots) \\
entre 5\% et 10\% & - & $23,7 \%(4707 / 19857)$ \\
entre 10\% et 15\% & 26 & $4,7 \%(934 / 19857)$ \\
entre 15\% et 20\% & 15 & $2,9 \%(579 / 19857)$ \\
entre 20\% et 25\% & 5 & $1,3 \%(262 / 19857)$ \\
\hline (évaluation la plus forte) & 5 & \\
\hline
\end{tabular}

\section{Tableau 1 \\ Évaluation moyenne de la performance communicationnelle des chefs pendant le débat}

Cette analyse de l'évaluation moyenne des électeurs en fonction de la production discursive des chefs a ainsi permis d'isoler cinq épisodes discursifs considérés comme étant les plus performants au cours du débat. Dans chacune des transcriptions de ces extraits, la production discursive correspondant à l'évaluation la plus élevée (entre $20 \%$ et $25 \%$ ) est indiquée en italique.

\section{Extrait 1. Débat des chefs (2004). Justice et enjeux sociaux}

Gilles Duceppe, chef du Bloc québécois, parti d’opposition

Monsieur Harper, on a toujours été responsables. Les seuls fronts communs organisés entre les partis d'opposition à Ottawa, c'est le Bloc qui les a organisés. Quand les libéraux ont eu besoin de nous autres sur le mariage gay, une chance que le Bloc était là. «(Harper:) Nous n'avons changé des choses: nous n'avons pas changé des choses:> Écoutez un instant. La démocratie, pour vous, est-ce que c'est seulement le pouvoir? «(Harper:) Non ce n'est pas:> Vous avez été dix ans dans l’opposition,

Par ailleurs, il faut considérer que l'évaluation de la performance d'un politicien par les électeurs touche à l'occasion une partie de la production discursive des autres politiciens lorsque ceux-ci manifestent des signaux d'écoute backchannel. 
est-ce que vous avez perdu votre temps? «(Harper:) Non c'est:〉 Moi, la démocratie, c'est de représenter ceux et celles qui nous font confiance, de défendre leurs intérêts, leurs valeurs, ce qu'ils veulent 〈(Harper:) Oui mais:〉 mais ce n'est pas de ma faute si ce que vous proposez ne correspond pas aux intérêts des Québécois. «(animateur:) Monsieur Harper〉 Je ne suis pas responsable de votre impuissance, moi.

\section{Steven Harper, chef du Parti conservateur}

Monsieur Duceppe, c'est important: c'est important de défendre des idées. Je fais ça depuis longtemps dans la politique. Mais, à la fin, on doit avoir des options pour le gouvernement et c'est ce que j'offre aux Québécois. «(animateur:) Merci〉

\section{Gilles Duceppe}

Mais les Québécois n’en veulent pas Monsieur Harper.

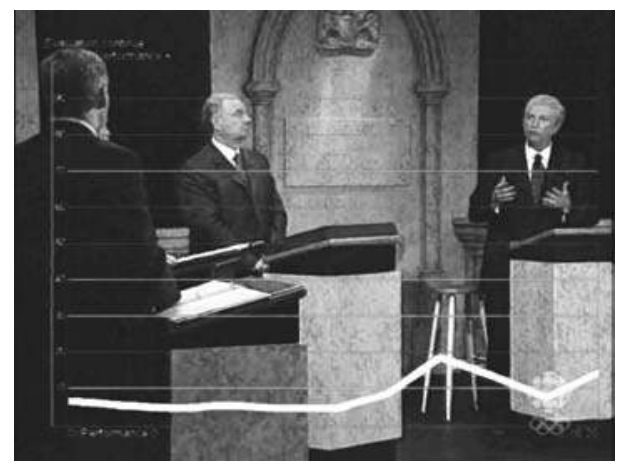

\section{Extrait 2. Débat des chefs (2004). Gouvernance et éthique}

Paul Martin, chef du Parti libéral, premier ministre sortant

Et s'il passe [si le Parti conservateur est élu], c'est un gouvernement qui n'aura aucun élu [au] Québec et qui a des philosophies à l'encontre des valeurs du Québec.

\section{Gilles Duceppe}

On a vu pendant dix ans Monsieur Martin ce que vous avez fait avec des élus du Québec hein. Dans la question des commandites là vous avez décidé à huis clos dans votre caucus de graisservos petits amis. Dans la question d'assurance-emploi vous avez détourné 45 milliards de la caisse d'assurance emploi. C'est ça que vous avez fait. Vous l'avez utilisée à d'autres fins. Même pour votre propre compagnie vous êtes intervenu <(Martin:) Non〉 pour changer les règles Monsieur Martin. C'est ça qu'on dénonce «(Martin:) Ça Monsieur:〉 et dans la question, je vais vous donner un exemple encore. 


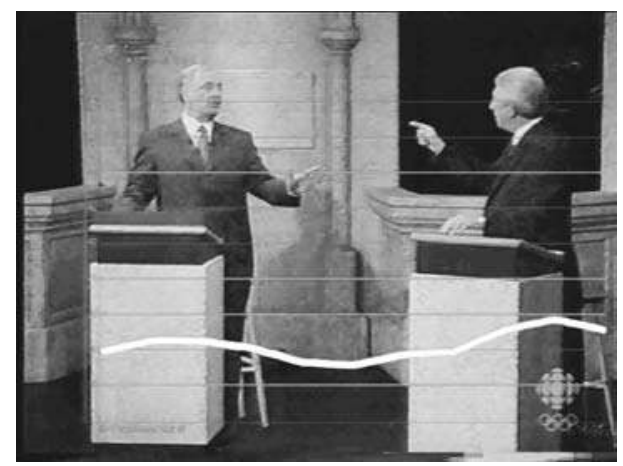

\section{Extrait 3. Débat des chefs (2004). Gouvernance et éthique}

\section{Gilles Duceppe}

Dans ce projet de loi, on parle de transport international maritime, de bateaux. CSL International c'est pas une compagnie de pédalos c'est une compagnie de bateaux ça. [Paul Martin, le premier ministre sortant, est propriétaire d'une compagnie de bateaux.] Et c'est de ça qu'on parle dans cette loi et on fait une rétroactivité parce que c'est en 1998 qu'elle est votée jusqu'en 1995. Or, joyeux hasard, en 1995, CSL International quitte le Liberia et arrive où? Aux Barbades! Devinez (rires) à quoi s'applique cette convention fiscale? Justement aux Barbades, la loi a été changée intentionnellement. Monsieur Martin nous dit ce soir c'est pas vrai. Je l'ai en main, je vous en passerai une copie tantôt. Vous étiez ministre des Finances, on parle de bateaux et on fait une rétroactivité que vous n'accordez pas aux personnes âgées pour le supplément de revenu garanti. Mais vous l'accordez à: 〈(Layton:) Nous sommes d'accord〉. «(animateur:) Monsieur Layton, Monsieur Layton〉. «(Layton:) Nous sommes d'accord`à Canadian Steamship International.

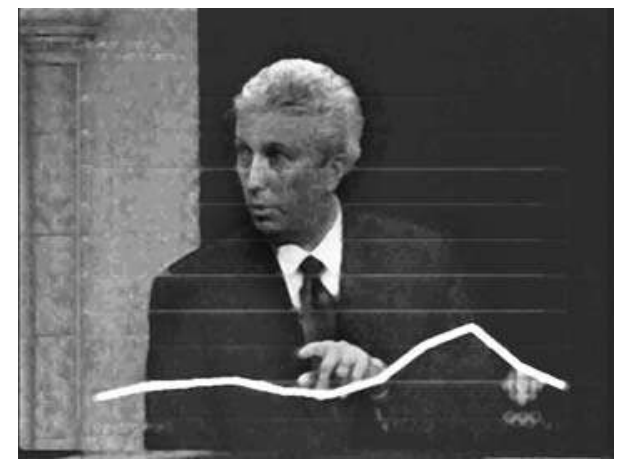




\section{Extrait 4. Débat des chefs (2004). Économie et finances}

\section{Paul Martin}

Comme vous le savez, la caisse d'assurance emploi, c'est une caisse qui est financée par le gouvernement canadien.

\section{Gilles Duceppe}

Je vous demande combien il y a dans la caisse?

\section{Paul Martin}

Et on a utilisé cet argent pour les congés parentaux.

\section{Gilles Duceppe}

Combien il y a dans la caisse, Monsieur Martin?

\section{Paul Martin}

Écoutez bien, la caisse a été établie par la vérificatrice générale.

\section{Gilles Duceppe}

Je vous demande combien? Donnez-moi un chiffre.

\section{Paul Martin}

Aujourd'hui, il y a assez d'argent.

\section{Gilles Duceppe}

Il y a zéro, il y a zéro, Monsieur Martin «(Martin:) Non, il n’a jamais été:〉 Il y a zéro, vous l'avez vidée, Monsieur Martin.

\section{Paul Martin}

Mais si je peux répondre, lorsqu'on a pris le pouvoir, il y avait un déficit.

\section{Gilles Duceppe}

Vous avez vidé la caisse!

\section{Paul Martin}

On ne vide pas un déficit, Monsieur Duceppe.

\section{Gilles Duceppe}

Vous avez vidé la caisse, 45 milliards de surplus, Monsieur Martin «(animateur:) Merci Vous ne voulez pas le dire «(Martin:) Non〉

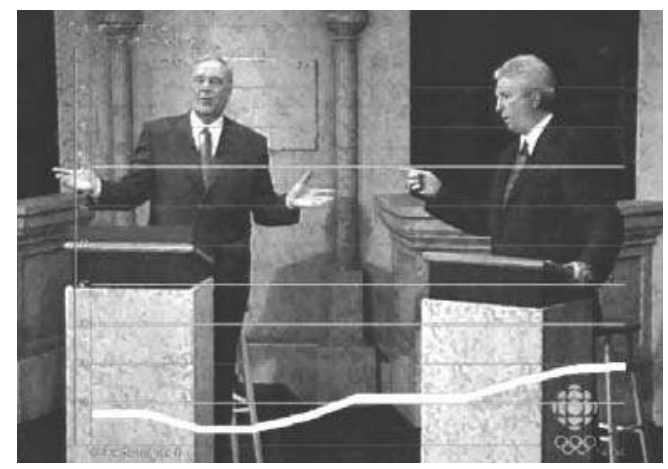




\section{Extrait 5. Débat des chefs (2004). Santé}

\section{Paul Martin}

Lavérité, c'est grâce aux sacrifices des Canadiens d'un océan àl'autre qu'aujourd'hui le Canada est en position d'investir dans la santé. «Duceppe: Le problème, c'est pas tous les Canadiens qui ont fait des sacrifices.〉〈animateur: Monsieur Duceppe, oui.〉 d'investir dans la santé.

\section{Gilles Duceppe}

Il y a plus d'enfants pauvres maintenant qu'il y en avait lorsque vous avez pris le pouvoir Monsieur Martin. Vous avez fait payer votre déficit par les chômeurs, vous avez pris 45 milliards dans la caisse d'assurance emploi. Vous avez fait payer votre déficit par les provinces puis par le Québec en coupant dans la santé et dans l'éducation. Ce que vous devriez dire au G8: "Je suis le seul pays du G8 qui a fait payer son déficit par les autres. » C'est ça que vous avez fait plutôt que de: «(Martin:) On est le seul:> Écoutez-moi, c'est à mon tour! «(animateur:) Dix secondes Monsieur Martin» plutôt que de couper dans la bureaucratie là.

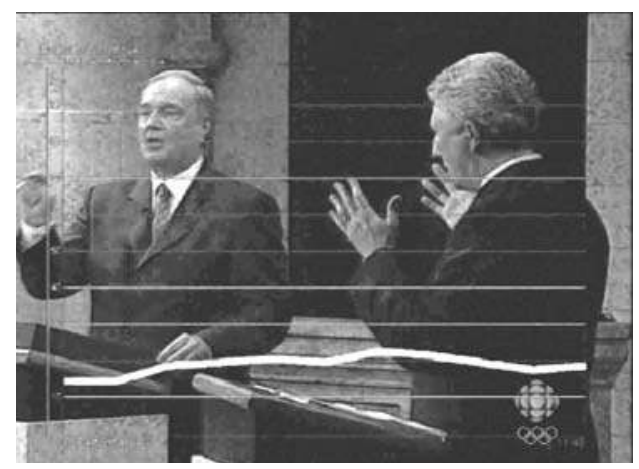

Les extraits précédents montrent que les cinq épisodes discursifs perçus par les électeurs comme étant les plus performants sont produits par le chef du Bloc québécois, Gilles Duceppe ${ }^{20}$. Cette évaluation, réalisée en temps direct par les électeurs, trouve écho dans les commentaires d'après débat des experts en communication qui ont unanimement reconnu la supériorité de la performance du chef bloquiste. À titre exploratoire et à la lumière des propositions théoriques et méthodologiques décrites précédemment, nous avons repéré quelques-uns des éléments de la stratégie de Duceppe qui ont pu contribuer à l'évaluation positive de sa performance ${ }^{21}$.

20. Une analyse plus fine isolant chacun des paramètres retenus pour l'étude montre que ce résultat est valide quelle que soit l'allégeance politique des informateurs.

21. Une analyse du discours des quatre chefs devra être réalisée sur l'ensemble du débat afin d'identifier les organisations de procédés discursifs qui participent à l'évaluation de leur performance communicationnelle respective. 
La première observation concernant ces cinq extraits est que le politicien se conforme discursivement à son identité professionnelle de chef d'un parti d'opposition en s'opposant, précisément, avec une certaine agressivité, ce qui est conforme aussi à la nature conflictuelle du genre télévisuel auquel correspond le débat politique 22 . Plusieurs expressions et procédés discursifs expriment une ferme opposition :

Extrait 1. Écoutez un instant. La démocratie, pour vous, est-ce que c'est seulement le pouvoir? [...] Vous avez été dix ans dans l'opposition, est-ce que vous avez perdu votre temps 23 ? [...] Mais ce n'est pas de ma faute si ce que vous proposez ne correspond pas aux intérêts des Québécois. [...] Je ne suis pas responsable de votre impuissance, moi.

Extrait 2. C'est ça qu'on dénonce.

Extrait 3. La loi a été changée intentionnellement. Monsieur Martin nous dit ce soir c'est pas vrai. Je l'ai en main, je vous en passerai une copie tantôt. Vous étiez ministre des Finances.

Extrait 4. Je vous demande combien il y a dans la caisse? [...] Combien il y a dans la caisse, Monsieur Martin? [...] Je vous demande combien? Donnez-moi un chiffre. Ily a zéro, il y a zéro, Monsieur Martin [...] Il y a zéro, vous l'avez vidée, Monsieur Martin. [...] Vous voulez pas le dire.

Extrait 5. Vous avez fait payer votre déficit par les chômeurs. Vous avez fait payer votre déficit par les provinces [...] Vous devriez dire au G8 [...].

L'extrait 1, considéré comme étant le plus performant selon l'évaluation des électeurs, consiste en une marque d'opposition forte fondée sur une incompatibilité (voir Perelman et Olbrechts-Tyteca, 1988, p. 264) entre le dire et l'agir du chef conservateur. Duceppe présente comme une contradiction le fait que Harper refuse de reconnaitre le rôle du Bloc québécois dans le parlement canadien sur la base de son impossibilité à former le gouvernement ${ }^{24}$, alors que luimême représente l'opposition officielle au Canada depuis dix ans. L'expression d'une telle contradiction discrédite le chef conservateur à plusieurs égards : elle révèle bien sûr une faille dans le contenu de son argumentation, mais elle fait surtout naitre une certaine suspicion autour des identités professionnelle et personnelle de Stephen Harper. La démocratie, pour vous, est-ce que c'est seulement le pouvoir? et Vous avez été dix ans dans l'opposition, est-ce que vous avez perdu votre temps? attirent l'attention des électeurs sur la personne

22. Voir Gingras, 2003.

23. La partie des exemples qui n'est pas en italique renvoie à des extraits de discours qui n'atteignent pas la zone de performance située entre $20 \%$ et $25 \%$. Toutefois, leur antériorité immédiate à cette zone nous permet de croire qu'ils contribuent à faire augmenter l'évaluation des électeurs. Rappelons que le décalage de deux secondes entre la production du discours et son évaluation requiert que nous prenions en considération certains de ces éléments.

24. Le Bloc québécois n'a pas de représentants à l'extérieur de la province de Québec. De ce fait, il est mathématiquement impossible pour ce parti de prendre le pouvoir au Canada. 
du politicien, sa conception de la démocratie et les raisons de son engagement en politique. La stratégie discursive de Duceppe favorise une construction du sens qui concerne la sincérité, voire l'intégrité, du chef Harper.

Dans l'extrait 3, Duceppe présente une autre opposition sous la forme d'une contradiction entre les dires du premier ministre Martin et une preuve tangible de son contraire sous la forme d'un document qu'il a en main. Bien qu'il reste implicite dans le discours, le sens que Duceppe permet au public électeur de construire à partir de ce procédé renvoie à un flagrant délit de mensonge pour le chef libéral. Dans les deux cas, 1 et 3, on peut comparer la stratégie discursive de Duceppe à un knock-out sur le plan communicationnel, puisque la contre-argumentation de la part des adversaires est à toute fin pratique impossible. L'évaluation de cette stratégie par les électeurs montre qu'elle permet de les ratifier assez unanimement.

Alors que l'affrontement direct et une certaine agressivité satisfont les attentes liées au genre télévisuel correspondant au débat et à l'identité professionnelle du chef d'un parti d'opposition, il faut savoir que la tolérance du public à l'égard de ce comportement connait des limites et que, à trop forte dose, la confrontation finit par agacer et mettre en danger la ratification du public. L'analyse des extraits précédents révèle que Duceppe ménage la sensibilité du public à cet égard en recourant régulièrement à l'ironie, à l'humour même, dans l'expression de son opposition.

Extrait 2. On a vu pendant dix ans Monsieur Martin ce que vous avez fait avec des élus du Québec hein.

Extrait 3. CSL International c'est pas une compagnie de pédalos c'est une compagnie de bateaux ça. [...] Or, joyeux hasard, en 1995, CSL International quitte le Liberia et arrive où? Aux Barbades! Devinez (rires) à quoi s'applique cette convention fiscale? Justement aux Barbades. [...] Je l'ai en main, je vous en passerai une copie tantôt.

Extrait 5. Ce que vous devriez dire au G8: "Je suis le seul pays du G8 qui a fait payer son déficit par les autres. »

L'ironie est un procédé particulièrement efficace pour ratifier, même à distance, les téléspectateurs. Dans le débat, un lien de connivence s'établit entre le politicien et les électeurs, sur la base d'un discours qui exclut les autres chefs. L'ironie resserre l'interaction entre eux, les réunissant dans un même univers de connaissances partagées. Au surplus, le ton ironique, à la limite humoristique pour ce qui est de l'extrait 3, procure au public un moment de répit dans une production sérieuse et formelle qui doit retenir leur attention pendant plus de deux heures. Comme le mentionne Tolson (2006, p. 81) au sujet du style de Tony Blair, "he did not seem to speak like a politician, but in a dialogical, colloquial and altogether "livelier" manner.»

Dans la même perspective de ratification du public, on note que Gilles 
Duceppe est celui qui, dans les cinq extraits précédents tout au moins, s’identifie le plus souvent au public des électeurs québécois. Il construit une relation privilégiée avec les Québécois, parmi lesquels il s’inclut et auxquels il s'adresse directement, et sur un ton parfois moins formel que les autres chefs, favorisant ainsi un sentiment d'appartenance.

Extrait 2. Vous avez décidé à huis clos dans votre caucus de graisser vos petits amis.

Extrait 3. Devinez (rires) à quoi s'applique cette convention fiscale? Justement aux Barbades, la loi a été changée intentionnellement. Monsieur Martin nous dit ce soir c'est pas vrai.

Extrait 3. CSL International c'est pas une compagnie de pédalos.

Dans l'extrait 1, l’identité personnelle de Duceppe, seul Québécois francophone de souche parmi les chefs, est clairement mise à profit pour ratifier le public privilégié auquel se destine le débat en français ${ }^{25}$ :

Extrait 1. Moi, la démocratie, c'est de représenter ceux et celles qui nous font confiance, de défendre leurs intérêts, leurs valeurs, ce qu'ils veulent.

Pris isolément, chacun de ces procédés ne saurait assurer le succès de la performance communicationnelle du chef du Bloc québécois. Si cela était, leur occurrence dans le discours des autres politiciens produirait la même réaction positive de la part des électeurs; or, les résultats de l'évaluation continue montrent que ce n'est pas le cas. Il n'y a pas de corrélation parfaite entre l'usage d'un procédé, aussi subtil soit-il, et la qualité de la performance communicationnelle. La performance d'un acte discursif résulte d'une stratégie d'ensemble, d'une organisation où chacun des procédés participe à la cohérence des autres.

Toutefois, les mécanismes de production du sens à la base des procédés relevés dans le discours de Duceppe semblent militer en faveur d'une conception interactionniste de la performance communicationnelle médiatique telle qu'elle a été proposée au début de cet article. Les recours à une variété de langue moins formelle, les adresses directes au public et l'appartenance au groupe des électeurs marquée par le pronom nous s’inscrivent avec cohérence dans une stratégie de ratification du public fondée sur une iden-

25. Lors des élections fédérales, le Débat des chefs a lieu à deux reprises : une fois en français pour un public majoritairement constitué d'électeurs francophones du Québec auxquels s'ajoutent les franco-canadiens des autres provinces, et une fois en anglais pour la majorité des électeurs anglophones de l'ensemble du Canada. Lors du débat en anglais, le lendemain, Gilles Duceppe a reçu la même meilleure performance de la part du public et des analystes, alors que l'anglais n'est pas, et de loin, sa langue maternelle, et qu'il est le chef d'un parti dont la vocation est de séparer le Québec du reste du Canada. Une analyse fine de la production discursive du chef bloquiste dans les deux versions du débat permettrait peut-être de montrer si l'identité personnelle est exploitée différemment. 
tité personnelle commune. Ces procédés contribuent à construire une personnalité publique moins distante, plus facilement assimilable aux individus qu'elle est censée représenter. Le message qui se crée au cours d'une telle interaction fait d'autant plus sens qu'il se construit entre des individus qui se reconnaissent aux mêmes champs expérientiels. Dans le genre conflictuel du débat, la mise en contradiction appelle une double construction du sens qui unit les interactants contre un adversaire commun : l'incompatibilité des faits révélée par le politicien amène le public à remettre en question non seulement les arguments mais la crédibilité de celui qui les produit. De ce point de vue, l'ironie constitue un procédé de ratification privilégié : pour exister, le sens de l'énoncé ironique requiert obligatoirement la coconstruction du public à partir d'un ensemble partagé de connaissances. Ainsi coconstruit, le sens de l'énoncé ironique resserre davantage le lien interactionnel qui unit ses coconstructeurs.

La conception interactionniste de la communication médiatique fait une distinction entre informer le public des évènements et créer du sens à partir des évènements dont le public est informé. L'un et l'autre objectifs requièrent une participation différente de la part du public, allant de récepteur à coconstructeur du sens. Le premier est mis au courant des évènements de l'actualité; le second interprète ces évènements dans l'ensemble des connaissances qui composent son univers pour produire du sens. L'évaluation de la performance communicationnelle du chef bloquiste pendant le Débat des chefs montre au surplus que le sens n'est pas une construction de nature exclusivement intellectuelle; il intègre de très nombreuses composantes émotionnelles.

L'interaction étant le lieu privilégié de la construction du sens, on peut conclure qu'une stratégie de communication médiatique efficace reposera sur une mise en scène constituée de procédés propres à ratifier le public dans le cadre d'une interaction non réciproque. Les recherches antérieures sur les journaux télévisés et l'analyse de la perception des électeurs au cours d'un débat politique télévisé donnent à penser que ces mises en scène visent tout spécialement à rejoindre le public dans son espace de réception. Cet objectif s'accomplit en simulant une interaction qui rappelle la communication interpersonnelle, tout en satisfaisant les attentes qui relèvent plus spécifiquement des expériences de communication médiatique des téléspectateurs. Une conclusion qui s'inscrit pleinement dans la pensée de Bakhtine (1977, p.41): "Tout signe, nous le savons, résulte d'un consensus entre des individus socialement organisés au cours d'un processus d'interaction. C'est pourquoi les formes du signe sont conditionnées autant par l'organisation sociale desdits individus que par les conditions dans lesquelles l'interaction a lieu.» 


\section{Références}

Amossy Ruth, 1999, Images de soi dans le discours, Lausanne, Delachaux et Niestlé.

- 2000, L'argumentation dans le discours, Paris, Nathan.

BAKHtIne Mikhail, 1977, Le marxisme et la philosophie du langage, Paris, Minuit.

Bıocca Frank A., DAVID Prabu, WeSt Mark, 1994, "Continuous response measurement (CRM). A computerized tool for research on the cognitive processing of communication messages», Measuring Psychological Responses to Media, A. Lang éd., Mahwah, Londres, Lawrence Erlbaum Associates.

BRoCK Franck, ReLIEU Marc, 1995, "L'infrastructure conversationnelle de la parole publique. L'analyse des réunions politiques et des interviews télédiffusées », Politix, ${ }^{\circ} 31$, p. 77-112.

BURGER Marcel, 2002, "Identities at stake in social interaction. The case of media interviews ", Studies in communication sciences, vol. 2, p. 1-20.

- à paraitre, "Les ethos typifiés et les ethos émergents comme condition de l'argumentation dans les médias», Análises do discurso hoje : ethos, émotions et argumentation, I. L. Machado, W. Emediato, R. de Mello éd., Rio de Janeiro, Editora Nova Fronteira.

Chanay (DE) Hugues, Kerbrat-Orecchioni Catherine, 2005, "100 minutes pour convaincre": l'ethos en action de Nicolas Sarkozy », Le français parlé des médias, Actes du colloque de Stockholm, M. Broth, M. Forsgren, C. Noren, F. Sulley-Nylander éd., p. 309-330.

Charaudeau Patrick, 1991, "Contrats de communication et ritualisations des débats télévisés», La télévision. Les débats culturels. Apostrophes, P. Charaudeau éd., Paris, Didier Érudition.

- 1997, Le discours d'information médiatique. La construction du miroir social, Paris, Nathan.

- 2005, Le discours politique. Les masques du pouvoir, Paris, Vuibert.

Charaudeau Patrick, Maingueneau Dominique, 2002, Dictionnaire d'analyse du discours, Paris, Le Seuil.

Coulomb-Gully Marlène, 2001, La démocratie mise en scènes. Télévision et élections, Paris, CNRS.

Drew Paul, Heritage John, 2006, Conversation Analysis, Londres, Sage.

FAIRCLAUgh Norman, 1995, Media Discourse, Londres, Oxford University Press.

FITCH Kristine L., SANDERS Robert E. éd., 2005, Handbook of Language and Social Interaction, Mahwah, Londres, Lawrence Erlbaum Associates.

GINGRAS Anne-Marie éd., 2003, La communication politique. État des savoirs, enjeux et perspectives, Québec, Presses de l'université du Québec.

Goffman Erving, 1981, Forms of Talk, Philadelphie, Philadelphia University Press.

HORTON Donald, WOHL Richard, 1986, « Mass communication and para-social interaction : observation on intimacy at a distance », Inter/media. Interpersonal Communication in a Media World, G. Gumpert, R. Cathcart éd., New York, Oxford University Press, p. 185-206.

HUTCHBY lan, 2005, «Conversation analysis and the study of broadcast talk», Hand- 
book of Language and Social Interaction, K. L. Fitch, R. E. Sanders éd., Mahwah, Londres, Lawrence Erlbaum Associates, p. 437-460.

- 2006, Media Talk. Conversation Analysis and the Study of Broadcasting, Maidenhead, Open University Press.

ISOTALUS Pekka, 1998, "Television performance as interaction», Nordicom Review, vol. 19, $\mathrm{n}^{\circ} 1$, p. 175-183.

Jost François, 1998, Penser la télévision, Paris, Nathan.

- 2007, Introduction à l'analyse de la télévision, Paris, Ellipses.

LARAMÉE Marie-Anne, 2003, "Le vedettariat journalistique à la télévision québécoise francophone», mémoire de maitrise, Université de Montréal.

LARrue Janine éd., Trognon Alain, 1994, "Les débats politiques télévisés», Pragmatique du discours politique, Paris, Armand Colin.

Livingstone Sonia, Lunt Peter, 1994, Talk on Television, Londres, New York, Routledge. Maingueneau Dominique, 1998, Analyser les textes de communication, Paris, Dunod.

MARTEL Guylaine, 1998, Pour une rhétorique du quotidien, Québec, Université Laval (CIRAL).

— 2004, «Humaniser les journaux télévisés. Les lieux privilégiés du journalisme d’interaction au Québec », Les Cahiers du journalisme, $n^{\circ} 13$, p. 182-205.

- 2007, "De la conversation dans l'information télévisée», Le français parlé des médias, Actes du colloque de Stockholm, M. Broth, M. Forsgren, C. Noren, F. SulleyNylander éd., p. 503-516.

- 2008, "Un point de vue interactionnel sur la communication médiatique », L'analyse linguistique des discours médiatiques. Entre sciences du langage et sciences de la communication, Colloque de Lausanne, M. Burger éd., Québec, Nota Bene, p. 113-133.

MARTEL Guylaine, TURBIDE Olivier, 2006, «Interagir avec le public. Quelques stratégies du discours médiatisé», Les interactions asymétriques, M. Laforest, D. Vincent éd., Québec, Nota bene, p. 49-64.

Olbrechts-Tyteca Lucie, Perelman Chaïm, 1970, Traité de l'argumentation. La nouvelle rhétorique, Bruxelles, Université de Bruxelles.

Politix. Revue des sciences sociales et du politique, 1997, n³7, Télévision et politique.

SCANnell Paddy éd., 1991, Broadcast Talk, Londres, Sage.

Scollon Ron, 1998, Mediated Discourse as Social Interaction. A Study of News Discourse, Londres, Longman.

Simons Herbert W., AghazARIAn Aram A., 1986, Form, Genre, and the Study of Political Discourse, Columbia, University of South Carolina Press.

Thompson John B., 1995, The Media and Modernity. A Social Theory of the Media, Cambridge, Polity Press.

Tolson Andrew éd., 2001, Television Talk Shows. Discourse, Performance, Spectacle, Mahwah, Londres, Lawrence Erlbaum Associates.

- 2006, Media Talk. Spoken Discourse on TV and Radio, Edinbourg, Edinburgh University Press.

VINCENT Diane, 2001, "Les enjeux de l'analyse conversationnelle ou les enjeux de la conversation », Revue québécoise de linguistique, vol. 30, nº 1, p.177-198. 